\title{
Bilateral Rectangular Musculocutaneous Advancement Flap: A Viable Alternative for Reconstruction of Large-Sized Forehead Defects with Exposed Bone
}

\author{
Hannara Park (D), Jaemin Seong (D), Hyouchun Park (D), Hyeonjung Yeo (D) \\ Department of Plastic and Reconstructive Surgery, Daegu Fatima Hospital, Daegu, Korea
}

\begin{abstract}
Reconstruction of large-sized forehead defects with exposed bone is challenging, as the forehead is an esthetically important area and the complex courses of the sensory nerves make them prone to damage during reconstruction. The purpose of this study was to achieve forehead reconstruction by predicting the pathways of the forehead sensory nerves and applying an operative technique that minimizes sensory nerve injury. We classified forehead sensory nerves that traverse the flap into five types according to their course, defined the frontalis muscle piercing line that allows for intuitive prediction of a nerve course, and devised a surgical technique for each nerve type. Next, we applied the techniques to a bilateral rectangular musculocutaneous advancement flap to reconstruct a large defect on the forehead caused by squamous cell carcinoma. No complications were observed except partial skin necrosis of the flap that healed spontaneously. Although tension and restricted early movement of the forehead was observed, it resolved within 2 weeks. After 5 months of treatment, the transverse scars were camouflaged by the skin creases and the sensory nerves of the forehead were intact.
\end{abstract}

Keywords: Forehead reconstruction; Supraorbital nerve; Supratrochlear nerve; Surgical flaps

\section{Introduction}

Skin defects on the forehead can result from tumors, trauma, congenital lesions, and burns and may vary in depth and size. Forehead reconstruction is challenging because of its aesthetic importance. It is especially difficult to achieve a satisfactory aesthetic outcome reconstructing large defects with exposed anatomical structures such as muscle, bones, and blood vessels, as they require a complex surgical technique [1-4].

For forehead reconstruction, surgeons can use various approaches including primary closure, skin grafting, local flaps, tissue expansion, regional flaps, and free flaps [1]. Local or distant flaps are appropriate for defects that expose anatomical structures such as muscles, periosteum, and bones following tumor resection [2]. Local flaps are preferred to skin grafts or regional/free flaps due to their superior color, texture, and functionality [5]. Musculocutaneous flaps are also a viable option, as they effectively cover deep defects that expose anatomical structures and have high flap mobility [6].

Many studies overlook the importance of preserving the sensory nerves of the forehead during reconstruction. They focus on camouflaging the scars formed over the healed defect and on producing an aesthetically satisfactory outcome. However, the forehead has many sensory nerve branches, which may traverse multiple skin layers

Case Report

Received: June 26, 2020

Revised: August 22, 2020

Accepted: September 7, 2020

Corresponding author:

Hannara Park, M.D.

Department of Plastic and Reconstructive Surgery, Daegu Fatima Hospital, 99 Ayang-ro, Dong-gu, Daegu 41199, Korea

Tel: $+82-53-940-7340$

Fax: +82-53-940-7344

E-mail: hannara_@hanmail.net

This is an Open Access article distributed under the terms of the Creative Commons Attribution Non-Commercial License (https://creativecommons.org/licenses/by-nc/4.0/) which permits unrestricted non-commercial use, distribution, and reproduction in any medium, provided the original work is properly cited.

(c) 2020 Korean Wound Management Society 
depending on their location in different forehead regions [1]. Due to the complex nerve anatomy of the forehead, surgeons could easily injure the sensory nerves, leading to sensory loss and discomfort.

Recent studies have described the course of the supraorbital nerve (SON) and the supratrochlear nerve (STN) in detail [79]. In the present study, we classified the forehead sensory nerves placed inside the flap into five types based on the flap location and the course of the nerves. We defined a frontalis muscle piercing line (FPL) to intuitively predict the courses of the nerves. Subsequently, we devised a surgical technique for each nerve type and applied the appropriate surgical techniques to a bilateral rectangular musculocutaneous advancement (BRMA) flap to reduce the risk of sensory nerve injury during forehead reconstruction. The study protocol conformed to the ethical guidelines of the Declaration of Helsinki, and verbal informed consent was obtained from the patient according to the decision of the Daegu Fatima Hospital Institutional Review Board (IRB exemption No. DFE20ORI072).

\section{Case}

A 70-year-old male patient underwent wide excision and a split-thickness skin graft on the forehead for treating squamous cell carcinoma. The patient developed a $3.4 \times 3.5 \mathrm{~cm}$ defect on the forehead that left the periosteum exposed and in an unhealthy state which caused skin necrosis. We therefore performed additional wide excision and musculocutaneous flap. With a safety margin of $0.4 \mathrm{~cm}$ applied to the defect at the 6 oclock position, the patient now had a $3.4 \times 3.9 \mathrm{~cm}$ defect located $3.5 \mathrm{~cm}$ superior to the supraorbital rim. The defect was located more toward the left side of the forehead. The distance between the medial portion of the left flap and the forehead midline was $2.1 \mathrm{~cm}$ and the distance between the medial portion of the right flap and the forehead midline was $1.3 \mathrm{~cm}$. The lengths of the upper and the lower borders of the designed flap were $3.9 \mathrm{~cm}$ each. The left and the right flaps were of the same size (Fig. 1A). Fig. 2 shows a schematic diagram of the nerve distribution in the forehead.

Wide excision was performed around the defect along the incision line and the periosteum was elevated using a periosteal elevator (Fig. 1B). The right flap was dissected on the lower border and two nerve branches were identified in the subcutaneous plane. No nerve branches were identified following the upper border dissection. The flap was dissected in the subfrontalis (subgaleal) plane and elevated to search for any nerve branches inside the flap. The soft tissue surrounding the nerve was dissected in the subcutaneous plane. We ensured that no excessive tension was applied to the nerve and there was no nerve injury following the advancement of the flap in the medial direction.

In the left flap, three nerve branches were identified in the subcutaneous plane of the lower border and one large branch was identified in the subfrontalis (subgaleal) plane, which was the most lateral plane. No nerve branches were identified following the upper border dissection. The flap was dissected in the subfrontalis (subgaleal) plane. A nerve was found traversing the lateral region of the flap. It was carefully dissected, completely separated from the flap, and placed in the periosteum. The nerve traversed a region of the flap lateral to the end of the upper border and entered the temporal region without piercing the flap. The flap was elevated and two nerve branches
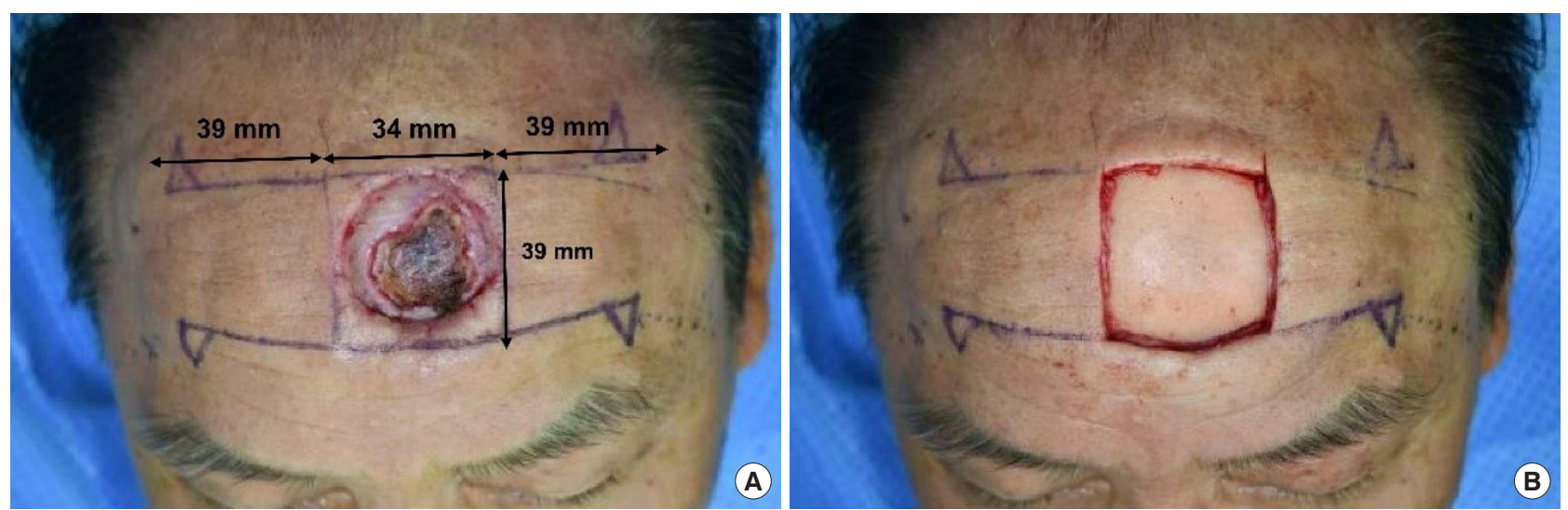

Fig. 1. The surgical procedure. (A) The defect was $3.4 \times 3.5 \mathrm{~cm}$ in size and the base of the defect was composed of unhealthy, exposed periosteum. (B) Wide excision was performed. The defect with bone exposure area was $3.4 \times 3.9 \mathrm{~cm}$ in size. 
Park H et al.

A viable alternative for forehead defect

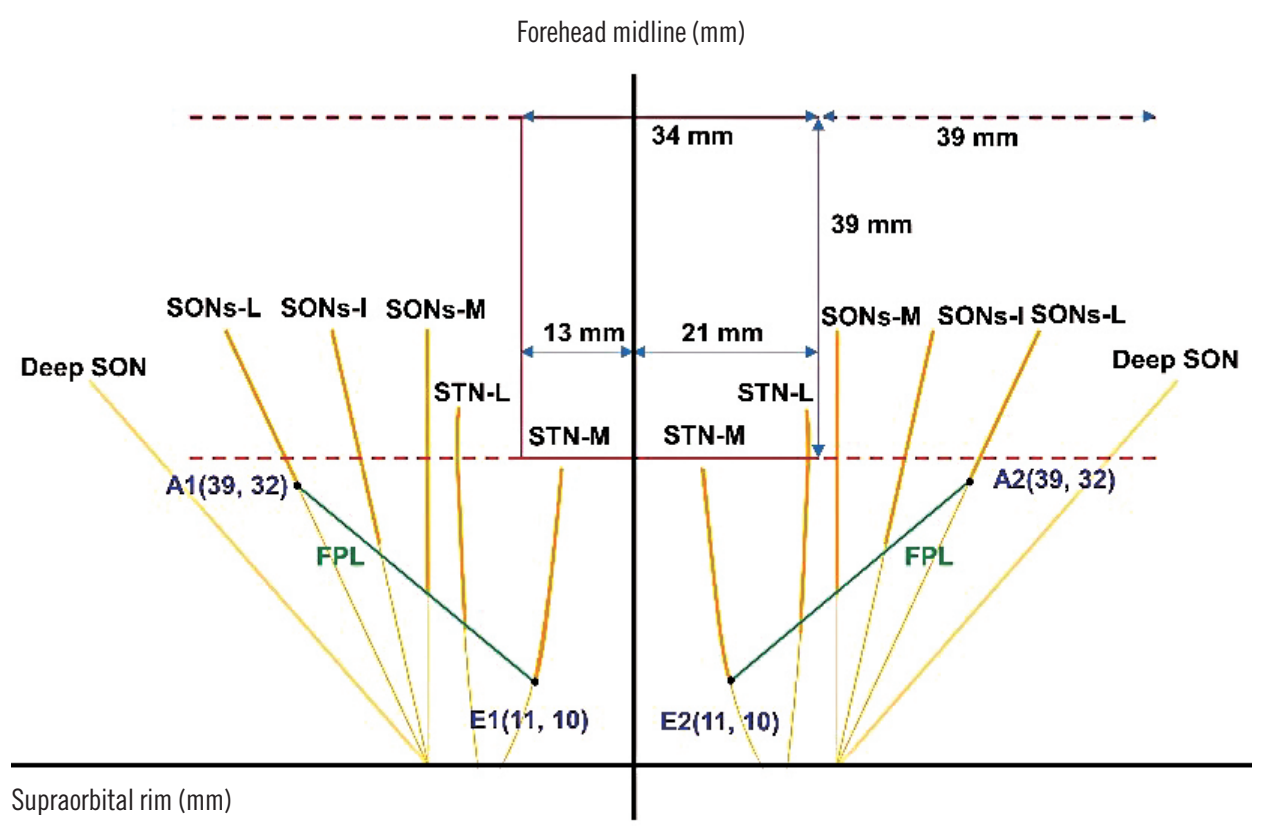

Fig. 2. A schematic diagram of the forehead defect and the nerve courses. SON, supraorbital nerve; SONs-I, intermediate branch of the superficial SON; SONs-L, lateral branch of the superficial SON; SONs-M, medial branch of the superficial SON; STN, supratrochlear nerve; STN-L, lateral branch of the STN; STN-M, medial branch of the STN; FPL, frontalis muscle piercing line.
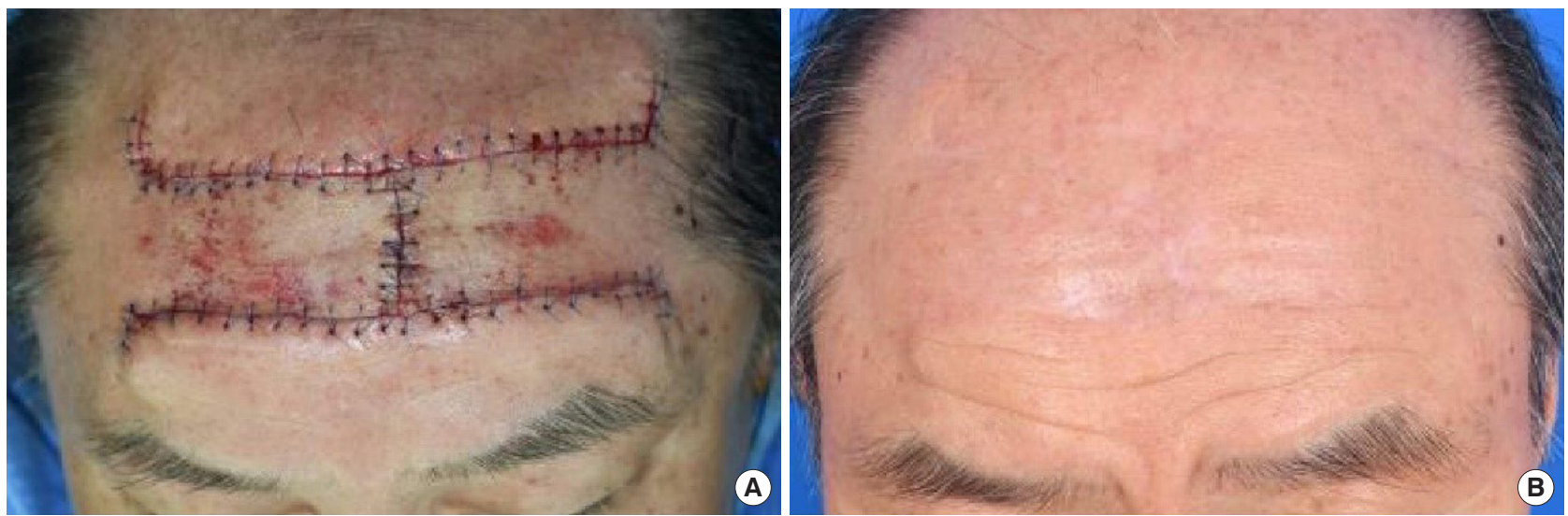

Fig. 3. Postoperative results. (A) Immediate postoperative photograph and (B) postoperative photograph after 5 months.

were located in the medial region of the flap. The soft tissues surrounding the branches were dissected in the subcutaneous plane. We ensured that no excessive tension was applied to the nerve and no nerve injury occurred while advancing the flap in the medial direction. Subsequently, the surgery was concluded (Fig. 3A).

There were no complications except partial skin necrosis of the flap that healed spontaneously. Although tension and restricted early movement of the forehead was observed, it resolved within 2 weeks. In the follow-up examination 5 months after the procedure, the transverse scars were camouflaged in the skin creases and the physical exam confirmed that the patient's forehead sensory was intact (Fig. 3B).

\section{Discussion}

The forehead is aesthetically important, as it occupies a large area on the face. Most of the studies on forehead reconstruction have focused on achieving excellent aesthetic outcomes. Nerve branches travel across multiple skin layers of the forehead following complex courses and are susceptible to injuries $[10,11]$. Any surgical method can be harmful for the patient if 


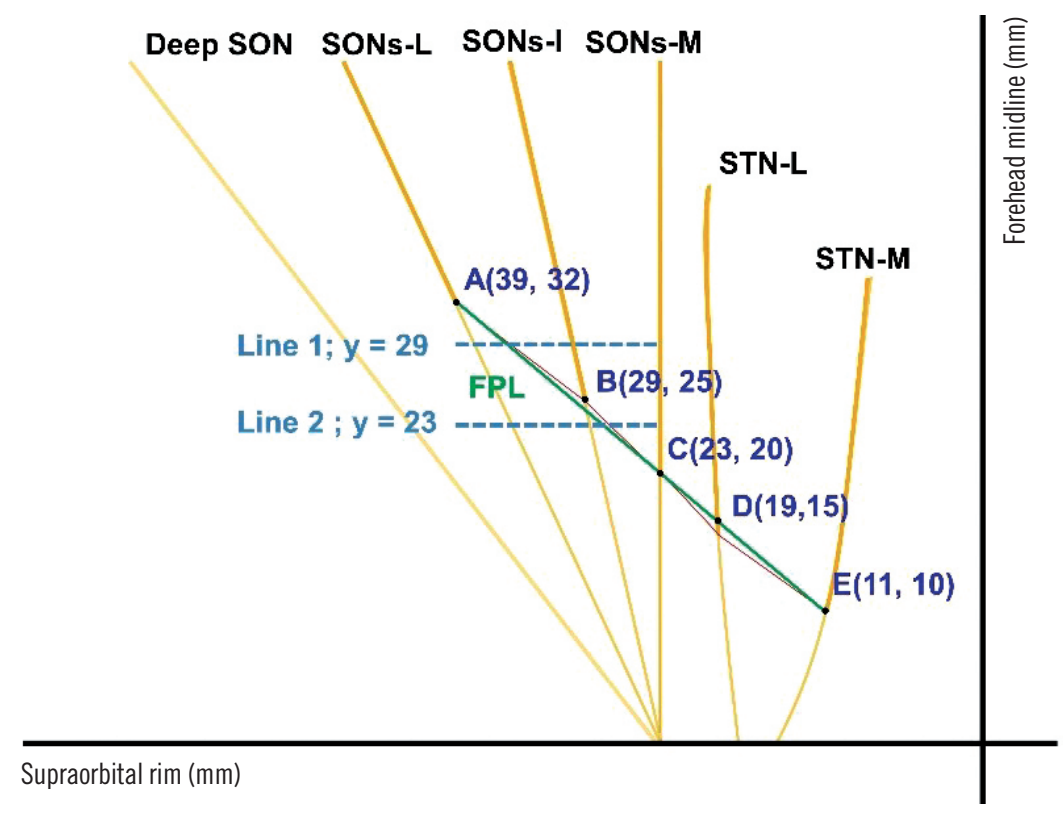

Fig. 4. A schematic diagram of the courses of forehead sensory nerves. A vertical line through the forehead midline and a horizontal line through the supraorbital margin were used as the horizontal and the vertical reference positions, respectively (horizontal and vertical dotted lines). The points and the lines are described in detail in the text (the section "Classification"). SON, supraorbital nerve; SONs-I, intermediate branch of the superficial SON; SONs-L, lateral branch of the superficial SON; SONs-M, medial branch of the superficial SON; STN, supratrochlear nerve; STN-L, lateral branch of the STN; STN-M, medial branch of the STN; FPL, frontalis muscle piercing line.

the surgeon does not have sufficient understanding of the forehead anatomy.

Studies have reported three superficial branches of the SON that pierce the frontalis muscle at various points. A previous study reported that the medial branch of the superficial SON (SONs-M) pierces the frontalis muscle at point $\mathrm{C}$, which is at mean horizontally $23 \mathrm{~mm}$ away from the forehead midline and vertically $20 \mathrm{~mm}$ away from the supraorbital rim. The intermediate branch of the superficial SON (SONs-I) pierces the muscle at point $\mathrm{B}$, which is at mean horizontally $29 \mathrm{~mm}$ from the forehead midline and vertically $29 \mathrm{~mm}$ from the supraorbital rim. The lateral branch of the superficial SON (SONs-L) pierces the muscle at point $\mathrm{A}$, which is at mean horizontally 39 $\mathrm{mm}$ from the forehead midline and vertically $32 \mathrm{~mm}$ from the supraorbital rim [7]. According to another study, these three branches of the SON pierce the frontalis muscle at a point located $26 \pm 3 \mathrm{~mm}$ superior to the supraorbital rim [9]. The two branches of the STN pierce the frontalis muscle at various points. The mean horizontal distance from the forehead midline and vertical distance from the supraorbital rim were 11 $\mathrm{mm}$ and $10 \mathrm{~mm}$ (point $\mathrm{E}$ in Fig. 4), respectively for the medial branch of the STN (STN-M) and $19 \mathrm{~mm}$ and $15 \mathrm{~mm}$ (point D in Fig. 4), respectively for the lateral branch of the STN (STN-
L) [8] (Fig. 4).

We defined the straight line connecting the points $\mathrm{A}$ and $\mathrm{E}$ as the FPL to predict the courses of the forehead sensory nerves. We hypothesized that the nerves in the region superior to the FPL pierce the frontalis muscle and are present in the subcutaneous plane. Fig. 4 is a schematic diagram showing the distribution of the forehead sensory nerves and the position of the FPL with respect to the supraorbital rim on the horizontal axis and the forehead midline on the vertical axis.

In the sagittal view of the flap cross-section after dividing the underlying muscles at the incision line, differences were observed among the nerve plane of the lower border, the plane traversed by the nerve within the flap, and the nerve plane of the upper border. We classified the forehead sensory nerves into five types based on the plane in which they were present and described a surgical technique appropriate for each nerve type. We created a novel technique, namely, the BRMA, which we believe is the most appropriate surgical technique for the nerve types aforementioned. We have presented our classification system in Tables 1 and 2.

By intuitively predicting the course of a nerve within the flap using the FPL, an effective flap design and operation plan could be developed and the operation time could be reduced. 
Park $\mathrm{H}$ et al.

A viable alternative for forehead defect

Table 1. Classification of the nerves in the flap

\begin{tabular}{llll}
\hline Classification & Nerve plane of the lower incision border & \multicolumn{1}{c}{ Plane traversed by the nerve within the flap } & Nerve plane of the upper incision border \\
\hline Type 1 & Subfrontalis (subgaleal) plane & Subfrontalis (subgaleal) plane & Subfrontalis (subgaleal) plane \\
Type 2 & Subfrontalis (subgaleal) plane & Subfrontalis (subgaleal) plane $\rightarrow$ subcutaneous & Subcutaneous plane \\
Type 3 & Subcutaneous plane & Subcutaneous plane & Subcutaneous plane \\
Type 4 & Subcutaneous plane & Subcutaneous plane & - \\
Type 5 & Subfrontalis (subgaleal) plane & Subfrontalis (subgaleal) plane $\rightarrow$ subcutaneous plane & - \\
\hline
\end{tabular}

Table 2. Classification of nerve sparing in BRMA flap reconstruction

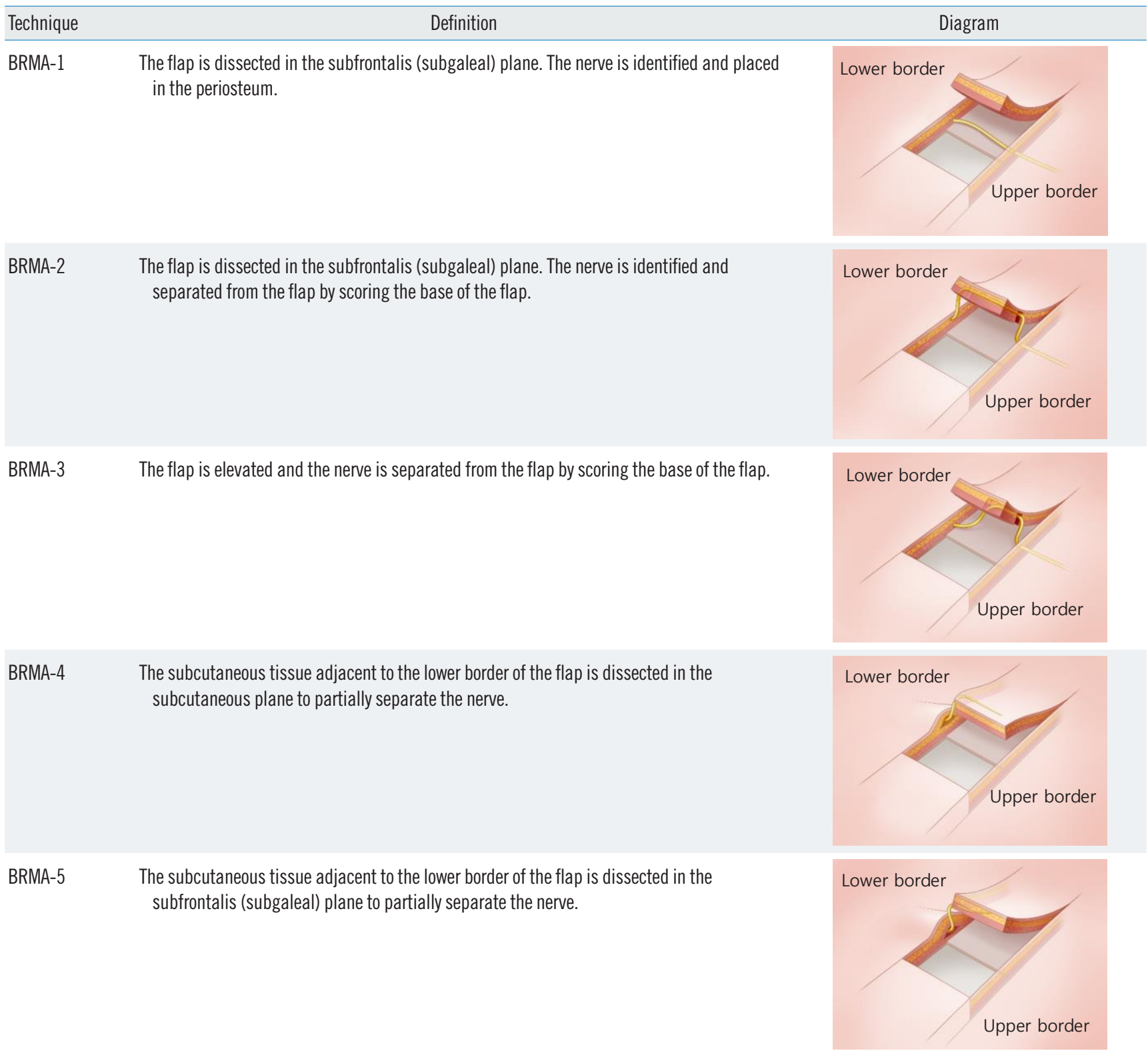

BRMA, bilateral rectangular musculocutaneous advancement.

Since a different technique is used for each nerve type, the surgical approach developed in this study can be applied to a flap containing multiple nerves. Different techniques may also be used for each of the bilateral flaps. In the present case, two type 
3 nerves were found in the right flap and two type 2 nerves and one type 1 nerve were found in the left flap. Based on these nerve types, BRMA-3 and BRMA-1 techniques were used for the respective flaps.

Our technique can be applied to flaps of different shapes. Once the points at which the nerve pierces a layer are identified, nerves can be successfully identified and separated from the flap regardless of the flap design. In our study, the deep SON in the left flap did not travel through the upper border but traveled in a more lateral direction. It therefore did not satisfy the criteria for type 1 nerve. However, we were aware of the layers involved in the course of the deep SON. We dissected the lower border and identified the nerve. Thus, we could separate the nerve traversing the flap in the subfrontalis (subgaleal) plane and place it in the periosteum.

While it is generally challenging for surgeons to achieve satisfactory functional and aesthetic outcomes after reconstructing forehead defects, a BRMA flap provides high aesthetic satisfaction, as it camouflages the scar along the incision line in the forehead wrinkles [10]. However, cases where lesions extend to more than $50 \mathrm{~cm}^{2}$ on the forehead are not suitable for reconstruction and making an incision and advancing the flap can cause nerve injuries. Most studies have overlooked the risk of such nerve injuries [3].

Other studies dealing with BRMA flaps have focused on methods that can effectively apply flaps, such as the appropriate defect size and length to width of flaps. In the present study, we were able to predict the course of a nerve in the flap using the FPL and classified nerve branches according to our nerve classification system. We successfully developed and applied operative techniques for different types of nerve branches to minimize nerve injuries and reconstructed large-sized forehead defects with exposed bone. We achieved excellent aesthetic outcomes without any sensory nerve injuries.

\section{Conflict of interest}

No potential conflict of interest relevant to this article was reported.

\section{ORCID iDs}

Hannara Park https://orcid.org/0000-0003-4158-0489
Jaemin Seong

Hyouchun Park

Hyeonjung Yeo

\section{References}

1. Boustany A, Ghareeb P, McClellan WT. Forehead reconstruction using a modified dual-plane A to T flap. Can J Plast Surg 2012;20:251-4.

2. Souza CD. Reconstruction of large scalp and forehead defects following tumor resection: personal strategy and experience-analysis of 25 cases. Rev Bras Cir Plast 2012;27: 227-37.

3. Kruse-Losler B, Presser D, Meyer U, et al. Reconstruction of large defects on the scalp and forehead as an interdisciplinary challenge: experience in the management of 39 cases. Eur J Surg Oncol 2006;32:1006-14.

4. Rocha LS, Paiva GR, de Oliveira LC, et al. Frontal reconstruction with frontal musculocutaneous $\mathrm{V}-\mathrm{Y}$ island flap. Plast Reconstr Surg 2007;120:631-7.

5. Guerrerosantos J. Frontalis musculocutaneous island flap for coverage of forehead defect. Plast Reconstr Surg 2000; 105:18-22.

6. Hallock GG, Trevaskis AE. Refinements of the subcutaneous pedicle flap for closure of forehead and scalp defects. Plast Reconstr Surg 1985;75:903-5.

7. Gil YC, Shin KJ, Lee SH, et al. Topography of the supraorbital nerve with reference to the lacrimal caruncle: danger zone for direct browplasty. Br J Ophthalmol 2017;101:9405.

8. Gil YC, Lee SH, Shin KJ, et al. Three-dimensional topography of the supratrochlear nerve with reference to the lacrimal caruncle, and its danger zone in Asians. Dermatol Surg 2017;43:1458-65.

9. Christensen KN, Lachman N, Pawlina W, et al. Cutaneous depth of the supraorbital nerve: a cadaveric anatomic study with clinical applications to dermatology. Dermatol Surg 2014;40:1342-8.

10. Fatah MF. Innervation and functional reconstruction of the forehead. Br J Plast Surg 1991;44:351-8.

11. Erdogmus S, Govsa F. Anatomy of the supraorbital region and the evaluation of it for the reconstruction of facial defects. J Craniofac Surg 2007;18:104-12. 